\title{
LXXXIII. The velocity of the ions of alkali salt vapours in flames
}

\author{
Prof. H.A. Wilson F.R.S. F.R.S.C.
}

To cite this article: Prof. H.A. Wilson F.R.S. F.R.S.C. (1911) LXXXIII. The velocity of the ions of alkali salt vapours in flames, Philosophical Magazine Series 6, 21:126, 711-718, DOI: $10.1080 / 14786440608637085$

To link to this article: http://dx.doi.org/10.1080/14786440608637085

册 Published online: 20 Apr 2009.

Submit your article to this journal $[\pi$

ЏII Article views: 4

Q View related articles $\asymp$

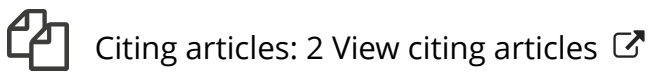


no longer constant, is the width of the lamina in the direction of motion at level $z$, we have

$$
\mathrm{F}=2 \rho(\nu / \pi)^{\frac{1}{2}} V^{\frac{3}{2}} \int b^{\frac{1}{2}} d z . \quad \cdot \quad \cdot .
$$

It will be seen that the result is not expressible in terms of the area of the lamina. In (49) $c$ is not constant, unless the lamina remains always similar in shape.

The fundamental condition as to the smallness of $v$ would seem to be realised in numerous practical cases ; but any one who has looked over the side of a steamer will know that the motion is not usually of the kind supposed in the theory. It would appear that the theoretical motion is subject to instabilities which prevent the motion from maintaining its simply stratified character. The resistance is then doubtless more nearly as the square of the velocity and independent of the value of $\nu$.

When in the case of bodies moving through air or water we express $V, a$, and $\nu$ in a consistent system of units, we find that in all ordinary cases $v / \mathrm{V} a$ is so very small a quantity that it is reasonable to identity $f(\nu / \mathrm{V} a)$ with $f(0)$. The influence of linear scale upon the character of the motion then disappears. This seems to be the explanation of a difficulty raised by Mr. Lanchester (loc. cit. $\$ 56$ ).

LXXXIII. The Velocity of the Ions of Alkali Salt Vapours in Flames. By Prot. H. A. Wilsox, F.R.S., F.K.S.C., McGill University, Montreal* .

T $\mathrm{T}$ was shown by the writer in $1899 \dagger$ that, in flames, all 1 the alkali metals give positive ions which have equal velocities due to an electric tield. This result has been confirmed by Marx and Moreau. The value of the velocity is about $70 \mathrm{cms}$. per sec. for one volt per cm.

The fact that the maximum quantity of electricity which can be carried hy a definite amount of any alkali salt vapour is equal to that required to electrolyse the same amount in a solution $\ddagger$, shows that the product $\mathrm{Ne}_{e}$ has the same value in salt vapours as in solutions. Here $N$ is the number of positive ions formed from one gram molecule of the salt when completely ionized and $e$ the charge carried by each ion. Since in solutions each atom of the alkali metal forms

* Communicated by the Author.

† Phil. Trans. A. cexxxvii. (1899).

† H. A. Wilson, Phil. Trans. A. cexcri. (1901). 
one monovalent positive ion, the result just mentioned makes it probable that the same thing happons in the vapours.

Prof. O. W. Richardson * has recently measured the ratio of the oharge $e$ to the mass $m$ for the positive ions of rapours of the sulphates of all the alkali metals, and tinds it equal to the value which obtains in solutions. This makes it very probable that the positive ions are metal atoms. The fact that the haloid salt vapours give ions having the same velocities as the oxysalts in flames, shows that all salts of any one metal give ions identical in nature.

The equality of the velocities of a lithium ion and a cæsium ion is difficult to explain on the view that they are simply singie atoms, for we should expect the velocity to depend on the atomic weight. The main object of this paper is to point a way out of this difficulty.

In my experiments two electrodes were placed one above the other in a Bunsen flame, and the current between them was measured. If the upper electrode was positively charged and a bead of salt was placed just below it, it was found that the current was not appreciably increased by the salt unless the potential difference between the electrodes was greater than about 100 volts. This was taken to mean that 100 volts was just enough to make the positive ions move down the flame. The potential gradient in the flame is nearly uniform except near the electrodes, so that the current density (i) is given by $i=e n\left(v_{1}+v_{2}\right)$, where $n$ is the number of ions of either sign per c.c., $v_{1}$ and $v_{2}$ the velocities of the positive and negative ions. If the gas is moving upwards with velocity $u$ and $\mathrm{X}$ denotes the electric intensity, then we have

$$
\begin{aligned}
& v_{1}=k_{1} \mathrm{X}-u \\
& v_{2}=k_{2} \mathrm{X}+u,
\end{aligned}
$$

so that the current is equal to en $\mathrm{X}\left(k_{1}+k_{2}\right)$, and is independent of $u$.

When the upper part of the flame is filled with salt vapour $n$ will be much larger in that part than in the rest of the flame, so that for a given current $X$ will be proportionally smaller. This diminution of $\mathrm{X}$, however, does not lead to an appreciable increase in the current, when the upper electrode is positive, because nearly all the resistance to the passage of the current is close to the negative electrode, where the greater part of the fall of potential takes place.

* Phil. Mag. Dec. 1910. 
Thus if $\mathrm{X}^{\prime}$ and $n^{\prime}$ refer to the upper part of the flame containing the salt, we have

$$
i=\mathrm{X}^{\prime} n^{\prime} e\left(k_{1}+k_{2}\right)=\mathrm{X} n e\left(k_{1}+k_{2}\right),
$$

which is independent of $u$, so that it does not appear at first sight why the salt should increase the current with any potential difference.

If, however, $\mathrm{X}$ is big enough, the metal ions will move down against the upward stream of gas and will be deposited on the negative electrode. The alkali metal will consequently accumulate at the lower electrode, and since it is strongly ionized it will diminish the resistance there and so increase the current. In the case of sodium salts this accumulation can be easily observed by the appearance of sodium light near the lower electrode*.

It appears, therefore, that the increase in the current is not as was originally supposed, due merely to the current carried by positive ions coming down, for before the salt is put in there are already present far more than enough ions to carry the current allowed by the resistance at the negative electrode.

Suppose that an alkali metal atom in the flame is ionized for a fraction $f$ of the time. Then its velocity due to an electric field will be $f k_{1} \mathrm{X}$ instead of $k_{1} \mathrm{X}$. If, then, $\mathrm{X}_{0}$ denotes the least value of $X$ for which the metal accumulates at the lower electrode, we have

$$
\begin{aligned}
f\left(k_{1} \mathrm{X}_{n}-u\right) & =(1-f) u, \\
f k_{1} & =\frac{u}{\mathrm{I}_{0}},
\end{aligned}
$$

for during the fraction $(1-f)$ of the time the atom will be carried upwards with velocity $u$.

The quantity which was determined experimentally was therefore not $k_{1}$ as was supposed, but $f k_{1}$. Now the conductivity imparted to a flame by equal numbers of molecules of different alkali metal salts increases rapidly with the atomic weight of the metal. This shows that a cæsium atom is ionized for a much larger fraction of the time than a lithium atom. Hence, since both give the same value for $f k_{1}$, it follows that $k_{1}$ for lithium must be really much greater than $k_{1}$ for casium.

In hot air at about $1000^{\circ} \mathrm{C}$. the $f$ 's will be much smaller

* H. A. Wilson, Proc. R. I. 1909.

Phil. Mag. S. 6. Vol. 21. No. 126. June 1911. 
than in a Bunsen flame, so that the values found for $f k_{1}$ should be much less, as was found to be the case*

The relative values of the fraction $f$ for different salts can be deduced from the conductivities which they impart to the flame. In the determination of $f k_{1}$ the concentration of the metallic atoms which move down the flame is extremely small, not enongh to appreciably colour the flame except in the case of sodium. The equilibrium between the atoms and negative electrons will, therefore, be determined by the equation

$$
q=\beta(\mathrm{N}-n)=\alpha n m, \quad . \quad . \quad .
$$

where $q$ is the number of positive ions produced per c.c. per sec. by ionization of the metal atoms, $N$ the total number of metal atoms present per c.c., $n$ the number of metal atoms which are ions per c.c., $m$ the number of negative electrons per c.c., and $\beta$ and $\alpha$ are constants. $m$ will be large compared with $n$ becanse the ions of the flame will be much more numerous than the ions from the metal vapour. Now $f=n / \mathrm{N}$, so that equation (1) becomes

$$
\beta(1-f)=a m f
$$

for another salt we have in the same way

Hence

$$
\beta^{\prime}\left(1-f^{\prime}\right)=\alpha^{\prime} m f^{\prime} \text {. }
$$

$$
\frac{\beta \alpha^{\prime}}{\alpha \bar{\beta}^{\prime}}=\frac{f}{f^{\prime}} \cdot \frac{1-f^{\prime}}{1-f} \text {. . . . . . . . }
$$

When the conductivities imparted to the flame by different salts were compared, the salts were present in comparatively large concentrations, and the number of ions due to the salts was large compared with the number due to the flame gases. In this case, therefore,

$$
q=\beta(\mathrm{N}-n),
$$

and for another salt having the same molecular concentration

$$
q^{\prime}=\beta^{\prime}\left(\mathrm{N}-n^{\prime}\right) \text {. }
$$

Here $n$ will be small compared with $\mathrm{N}$, so that approximately

Then (2) and (3) give

$$
\frac{q}{q^{\prime}}=\frac{\beta}{\beta^{\prime}} \cdot \text {. . . . . . . }
$$

$$
\frac{q \alpha^{\prime}}{q^{\prime} \alpha}=\frac{f}{f^{\prime}} \cdot \frac{1-f^{\prime}}{1-f^{\prime}} . . . . . .
$$

* H. A. Wilson, Phil. Trans. A. cexxxvii. (1899). 
It will be observed that in this equation the $f$ 's apply to very small concentrations, while the $q$ 's are for a comparatively large concentration.

Sir J. J. Thomson has given the theory* of the relation between the potential difference $(V)$ between two electrodes in a flame and the current (i). He obtains the equation

$$
\mathrm{V}=\left(\frac{\alpha}{q}\right)^{\frac{1}{2}} \frac{1}{e k_{2}}\left(l i+\frac{\alpha i^{2}}{4 \pi e^{2} q \sqrt{k_{1} k_{2}}}\right) \text {. }
$$

When the electrodes are near together, as was the case in the measurements of the currents with different salts, the first term can be neglected, so that

$$
\mathrm{V}=\frac{i^{2} \alpha^{\frac{3}{2}}}{4 \pi e^{3} k_{2^{3}}^{\frac{3}{3}} \overline{k_{1}}{ }^{\frac{1}{2}} y^{\frac{3}{2}}} .
$$

If $i^{\prime}$ denotes the current obtained with another salt and the same $\mathrm{V}$, then

$$
\left(\frac{i}{\bar{i}^{\prime}}\right)^{2}=\left(\frac{\alpha^{\prime} q}{\alpha g^{\prime}}\right)^{\frac{3}{2}}\left(\frac{k_{1}}{k_{1}^{\prime}}\right)^{\frac{1}{2}}
$$

The experiments show that $f^{\prime} k_{1}=f^{\prime} k_{1}^{\prime}$. Hence, using (4),

$$
\begin{aligned}
\left(\frac{i}{i^{\prime}}\right)^{2} & =\left(\frac{f}{f^{\prime}} \cdot \frac{1-f^{\prime}}{1-f}\right)^{\frac{3}{2}}\left(\frac{f^{\prime}}{f^{\prime}}\right)^{\frac{1}{2}}, \\
\text { or } \quad \frac{i}{i^{\prime}} & =\left(\frac{f}{f^{\prime \prime}}\right)^{\frac{1}{2}}\left(\frac{1-f^{\prime}}{1-f^{\prime}}\right)^{\frac{3}{4}} . . . . . .
\end{aligned}
$$

Here $i$ and $i^{\prime}$ are the currents given by the same P.D. with two different salt vapours of equal concentration. The ratio $i / i^{t}$ ought therefore to be independent of the concentration. When the concentration of the salt is not too large this is true, for then the current is proportional to a power of the concentration, which is about one-half for all salts.

The following table $\dagger$ gives the currents observed when a $\frac{1}{10}$ normal solution of the chloride was sprayed into the flame

* 'Conduction of Electricity through Gases,' 2nd ed.

† "The Electrical Conductivity and Luminosity of Flames containing Vaporized Salts," Smithells, Dawson, and Wilson: Phil. Trans. A. cexli. (1899). 
using a P.D. of $5 \cdot 60$ volts. With this P.D., the first term in Sir J. J. Thomson's equation can be safely neglected.

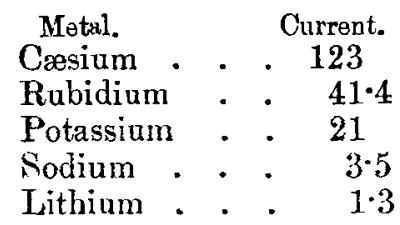

In order to use these values of the currents to calculate the $f^{\prime \prime}$ 's we require another relation. If the positive ions consist of single atoms, then their velocities ought to be approximately inversely proportional to the square roots of their atomic weights (M), consequently $f$ ought to be proportional to $\sqrt{\mathrm{M}}$.

Instead of (5) we can write

$$
i=\frac{\mathrm{A} f^{\frac{1}{2}}}{(1-f)^{\frac{3}{4}}}
$$

where $\mathrm{A}$ is a constant. Putting $f=\mathrm{B} \sqrt{\mathrm{M}}$ this becomes

$$
i=\frac{\mathrm{AB}_{\frac{1}{2}} \mathrm{M}^{\frac{1}{4}}}{\left(1-\mathrm{B}^{\frac{1}{2}}\right)^{\frac{3}{4}}} \text {. }
$$

Two values of $i$ and $M$ then suffice to determine $A$ and $B$. Using the values for cæsium and sodium gives $\mathrm{B}=0.08594$ and $A=3 \cdot 67$.

With these values of $\mathrm{A}$ and $\mathrm{B}$ we get the values of $f$

\begin{tabular}{|c|c|c|c|c|}
\hline Metal. & $f$ & $f^{\prime}$. & $k_{1}$ & $k_{1}^{\prime}$. \\
\hline Crebium ....... & 0.99 & 099 & 71 & 71 \\
\hline Rubidium ,........... & 079 & 0.96 & 89 & 73 \\
\hline Potassium........ & $0 \cdot 54$ & 091 & 130 & 77 \\
\hline Sodium ............... & $0 \cdot 41$ & 041 & 170 & 170 \\
\hline Lithium.............. & 0.23 & $0 \cdot 21$ & 305 & 333 \\
\hline
\end{tabular}
given in the second column of the following table :-

The column headed $f^{\prime}$ contains the values of $f$ required by the observed currents. The differences between $f$ and $f^{\prime}$ are not very great, except in the case of potassium. It seems, therefore, that the assumption that $f$ varies as $\sqrt{\mathrm{M}}$ is roughly true. The column headed $k_{1}$ contains the values of the velocities of the positive ions got by using the numbers for $f$, and that headed $k_{1}{ }^{\prime}$ those corresponding to the numbers 
under $f^{\prime}$. The value of $f k_{l}$ was taken to be $70 \mathrm{cms}$. per sec.

The value of $k_{1}$ can be calculated roughly on the kinetic theory of gases, for in a flame at about $2000^{\circ} \mathrm{C}$. the free path $(\lambda)$ of an atom is probably about $10^{-4} \mathrm{~cm} .^{*}$ The well known formula $k_{1}=e \lambda / m \mathrm{~V}$ gives $k_{1}=300 \mathrm{cms}$. per sec. for an atum of hydrogen, or 120 for an atom of lithium. This is about one-third the value of $k_{1}$ as estimated above, which is as near as could be expected.

I think, therefore, that the evidence provided by the measurements made with the object of finding the velocities of the positive ions in flames is not inconsistent with the view that these ions are single atoms of the alkali metal.

Measurements on the negative ions in flames have also been made by the writer and others $t$. The negative ions appear to be free electrons, so that their deposition on the positive electrode cannot be supposed to cause an increase in the current, as in the case of the positive ions at the negative electrode. It seems, therefore, that the supposed determinations of the velocity of the negative ions in flames, by finding the least P.D. required to make them move against or across the stream of gas, are based on a fallacy. When the salt is put in near the negative electrode the large resistance there is diminished, so that the current onght to be increazed, whether the P.D. is big enough to make the negative ions move against the stream or not. The following table contains the currents observed taken from my pape: $\ddagger$.

\begin{tabular}{|c|c|c|}
\hline \multirow{2}{*}{$\begin{array}{l}\text { P.D. } \\
\text { (Volts). }\end{array}$} & \multicolumn{2}{|c|}{ Current. } \\
\hline & (Without salt.) & (With salt.) \\
\hline 0 & -3 & -13 \\
\hline $0 \cdot 25$ & -2 & -10 \\
\hline 0.5 & 0 & -7 \\
\hline 075 & +2 & 0 \\
\hline $1 \cdot 0$ & +3 & +5 \\
\hline 15 & +3 & +20 \\
\hline 20 & +3 & +30 \\
\hline 30 & +3 & +33 \\
\hline
\end{tabular}
2nd ed.

* Sir J. J. Thomson, 'Conduction of Electricity through Gases,'

+ Marx, Moreau, and E. Gold.

$\ddagger$ Phil. Trans. A. cexxxvii. p. 517 (1899). 
The increase in the current between one and two volts with salt below the upper (negative) electrode was supposed to sbow that above one volt the negative ions from the salt moved down the flame. The increase in the current below one volt is however quite marked. It was then supposed that the flams without salt was not strongly ionized, but now it is known that the small current without salt is due to the great resistance close to the negative electrode, and that the ions present are sufficient to carry a current of probably many amperes. Such experiments, therefore, do not give any information with regard to the velocity of the negative ions.

Under these circumstances it is necessary to fall back on indirect evidence. Measurements of the effect of a magnetic field on the conductivity of a Bunsen flame made by the writer ${ }^{*}$ indicated that the velocity of the negative ions was about $9000 \mathrm{cms}$. per sec., which is about the value to be expected for negative electrons.

We may therefore conclude that the positive ions of alkali salts in flames are probably single atoms of the metal, and that the negative inns are electrons.

In a recent paper $\mathrm{Mr}$. Lusby $\dagger$ finds $290 \mathrm{cms}$. per sec. for the velocity of the positive ions of salt vapours in flames. In his experiments the electrodes were only $3 \mathrm{cms}$. apart, so that in the absence of salt the uniform gradient was not present because the negative drop extends more than $2 \mathrm{cms}$. from the lower electrode. On putting in the salt near the upper electrode he observed a very small uniform gradient which is evidently due to the high conductivity of the salt vapour. To calculate the velocity of the ions correctly the value of the uniform gradient just below the salt vapour is required, and this should be equal to the gradient in the absence of salt since the current is unchanged at the critical potential. I think therefore that Mr. Lusby's result is too high.

LXXXIV. The Number of Electrons in the Atom. By Prof. H. A. WIJson, F.R.S., F.R.S.C., McGill Lniversity, Montreal $\ddagger$.

A CCORDING to Sir.J.J. Thomson's theory § atoms may $A$ be regarded as spheres of positive electricity containing negative electrons which can move about freely inside the positive charge. The total negative charge on the electrons

* Proc. Roy. Soc. A., vol. lxxxii.

† Proc. Camb. Phil. Soc, vol. xvi. Pt. 1, 1911.

\pm Communicated by the Author.

$\S$ 'The Corpuscular Theory of Matter,' 1907. 